\title{
Intervenciones neuroquirúrgicas en Cataluña. Resultados de una encuesta anes- tésica durante 2003
}

\author{
P. Hurtado; R. Valero; S. Fernández; A. Alcón; N. Fábregas y Grupo ANESCAT*
}

Servicio de Anestesiología y Reanimación Hospital Clinic de Barcelona. *Societat Catalana d'Anestesiologia, Reanimació i Terapéutica del Dolor

\section{Resumen}

Introducción. La planificación sanitaria requiere el conocimiento preciso de la actividad desarrollada. Presentamos los resultados de la actividad neuroanestésica en Cataluña en 2003.

Material y métodos. Encuesta prospectiva y transversal realizada en 14 días aleatorizados del 2003. Participaron todos los hospitales que practicaban anestesia en Cataluña. Se incluyeron datos sobre las características de los pacientes, técnicas anestésicas y tipo de procedimiento.

Resultados. Se recogieron un total de 265 procedimientos neuroanestésicos, representando el $1,28 \%$ del total de cirugías. Los datos permiten extrapolar que se realizaron 6.909 procedimientos neuroanestésicos en toda Cataluña durante el año 2003 (95\% IC 60227847). El $74 \%$ de los procedimientos se realizaron en hospitales públicos y el $26 \%$ en hospitales privados. La edad media de los pacientes fue de 48 años (IC 95\% 45,5-50,6). Los procedimientos programados fueron el $79,7 \%$. Las intervenciones más frecuentes fueron:

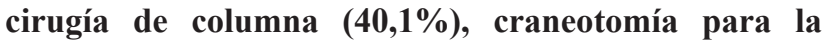

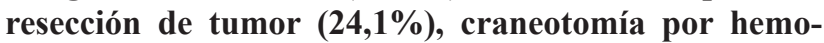
rragia o trauma $(7,2 \%)$, craneotomía para clipaje de aneurisma o cirugía de malformación arterio-venosa (MAV) $(2,3 \%)$ y la derivación ventricular $(2,6 \%)$. La duración media de las craneotomías para resección de tumor fue de $287 \pm 95 \mathrm{~min}$. Los pacientes fueron controlados postoperatoriamente en una sala de recuperación convencional $(55,8 \%)$, en una unidad de cuidados monitorizado $(19,2 \%)$ o en una unidad de cuidados intensivos $(\mathbf{2 4 , 9 \% )}$ ). Los pacientes tras craneotomía fueron admitidos principalmente en una unidad de cuidados intensivos $(41,6 \%)$ o en una unidad de vigilancia monitorizada $(33,7 \%)$.

Conclusiones. Alrededor de siete mil procedimientos neuroanestésicos fueron realizados en Cataluña en el 2003, el $74 \%$ de los cuales se realizaron en hospitales

Recibido: 29-11-06. Aceptado: 6-06-07 públicos y el $26 \%$ en hospitales privados. La cirugía de columna y la craneotomía para resección de tumores fueron las intervenciones más frecuentes. Los pacientes con craneotomía fueron remitidos principalmente a la UCI o a unidades de vigilancia monitorizada en el postoperatorio inmediato.

PALABRAS CLAVE: Neuroanestesia. Cataluña. Procedimientos. Encuesta

Neuroanesthetic practice in Catalonia. Results of a survey done on 2003

\section{Summary}

Introduction. Health system planning requires the precise knowledge of the activity performed. We present the neuroanesthesic activity results in Catalonia during 2003.

Methods. A prospective and cross-sectional survey was performed for 14 randomised days during 2003. All hospitals practicing anaesthesia in Catalonia took part in the survey. Data on characteristics of patients, anaesthetic techniques and type of procedure were included.

Results. 6909 neuroanesthetic procedures performed in Catalonia in 2003 (95\% IC 6022-7847), a $1.28 \%$ of total surgical activity. A $74 \%$ of procedures were done in the public hospitals and a $26 \%$ in private hospitals. Patients mean age was 48 years old (95\% IC 45.5-50.6). Scheduled procedures were $\mathbf{7 9 . 7 \%}$. The most frequent interventions were: Spine surgery $40.1 \%$; cranioto-

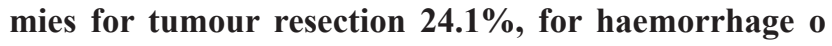
trauma $7.2 \%$ and for aneurysms or AVM surgery $2.3 \%$;

Abreviaturas. AANS: Asociación Americana de Cirujanos Neurológicos. ANESCAT: actividad anestésico-quirúrgica realizada en todos los hospitales de Cataluña. ASA: Sociedad Americana de Anestesiología. IC: intervalo de confianza. MAV: malformación arteriovenosa. SCARDT: Societat Catalana de Anestesiología, Reanimació i Terapèutica del Dolor. UCI: Unidad de cuidados intensivos. 
ventricular shunts $2.6 \%$. Mean duration of craneotomies for tumour resection was $287 \pm 95 \mathrm{~min}$. All patients were distributed postoperatively in a conventional recovery room $(55.8 \%)$, in a monitored care unit $(19.2 \%)$ or in an intensive care unit (24.9\%). Craniotomy patients were admitted to an intensive care unit $(41.6 \%)$, a monitored care unit $(33.7 \%)$ or a conventional recovery room $(24.7 \%)$.

Conclusions. About seven thousand Neurosurgical procedures were performed in Catalonia in 2003, in public $(\mathbf{7 3 . 6 \%})$ and private $(\mathbf{2 6 . 4 \% )}$ hospitals. Spine surgery and craniotomies for tumour resection were the most frequently performed interventions. Craniotomy patients were mainly submitted to an ICU or a Monitored care unit.

KEY WORDS: Neuroanaesthesia. Catalonia. Procedures. Survey.

\section{Introducción}

La planificación sanitaria, y en nuestro caso, la planificación de las necesidades sanitarias anestésicas requiere el conocimiento preciso de la actividad que se desarrolla en nuestro medio. En 2003 se llevó acabo una encuesta sobre la actividad anestésico-quirúrgica realizada en todos los hospitales de Cataluña (ANESCAT) ${ }^{7}$. A diferencia de otros países, hasta ese momento no disponíamos de datos contrastados que proporcionaran información sobre la globalidad de procedimientos anestésico quirúrgicos practicados en nuestro medio. La "Societat Catalana de Anestesiologia, Reanimació i Terapéutica del Dolor" (SCARDT) diseñó esta encuesta para poder conocer con precisión los tipos de procedimientos realizados con mayor frecuencia en Cataluña, así como la técnica anestésica utilizada y las características demográficas de la población intervenida. Este estudio es similar a otros realizados entre 1996 y 1999 en Francia e Italia ${ }^{3,6}$.

Un grupo de anestesiólogos, especialmente dedicados a la neurocirugía, decidimos analizar las características de la actividad neuroquirúrgica a partir de los datos recogidos en la encuesta general. En los últimos años, la neurocirugía ha experimentado cambios debidos al aumento de las prácticas diagnósticas e intervencionistas, al desarrollo de nuevas tecnologías y al uso cada vez mayor de técnicas loco-regionales. Además de los cambios debidos a la mejoría tecnológica y farmacológica también se ha encontrado un aumento en la media de edad de la población que es intervenida y por tanto un aumento de la patología concomitante. Estos datos son los primeros disponibles en nuestra área y pueden ser de utilidad como punto de partida para futuros estudios epidemiológicos.

\section{Pacientes y métodos}

La encuesta ANESCAT fue realizada por la "Societat Catalana de Anestesiologia, Reanimació i Terapéutica del Dolor" (SCARTD), con la participación de todos los centros donde se realizaba actividad anestésica en Cataluña. Participaron 131 centros entre públicos y privados, el $100 \%$ de los que practicaban anestesia en Cataluña en ese momento.

Se planificó un estudio prospectivo transversal en forma de encuesta incluyendo 14 días de corte a lo largo del 2003 (dos días por cada día de la semana) siendo los mismos días para todos los centros.

Se elaboró un cuestionario individualizado que recogía las características demográficas de los pacientes anestesiados, de la técnica anestésica y del tipo de intervención. Se calculó la representatividad de la población calculando un riesgo alfa de 0,05 . Se estimó la tasa de anestesias como una proporción estimada del $50 \%$.

Se tuvo en cuenta la estratificación por sexo, grupos de edad y puntuación en la escala de valoración del riesgo anestésico de la Sociedad Americana de Anestesiología (ASA). El mínimo de cuestionarios necesarios para obtener una muestra aleatoria poblacional fue de 12.288 asumiendo que la población era de 6.343.110 habitantes (censo del año 2001). La estimación oficial de intervenciones quirúrgicas en Cataluña en el año 2001 fue de 350.000. Según esta cifra, para obtener la muestra calculada se debía recoger la actividad de 12,8 días del año. Para asegurar el número mínimo de anestesias a recoger, la encuesta se diseñó para realizar 14 cortes de 24 horas del año 2003 y estos 14 días fueron escogidos de manera aleatoria. Para mantener la motivación y el rigor de la recogida de datos, se realizaron sesiones informativas en los diversos centros.

Tras realizar una revisión de la homogeneidad de los datos estos fueron introducidos en una base de datos confeccionada en MS Access (Microsoft Corp. ${ }^{\circledR}$ ) por dos introductores de datos. Cada 2.000 cuestionarios introducidos se realizaba un control de calidad. Si se detectaban inconsistencias se corregían solicitando información a los centros participantes ${ }^{7}$. El análisis estadístico se llevó a cabo con el paquete estadístico SPSS 11.0 (SPSS Inc. $\left.{ }^{\circledR}\right)$. Para la estadística descriptiva se usaron frecuencias absolutas y relativas, medias, medianas, rangos, percentiles e intervalos de confianza (IC) del 95\% de las medias, medianas y tasas. Dado el bajo número de datos perdidos se asumió que estos se comportaban de la misma manera que los valores conocidos.

Se incluyeron datos sobre las características de los pacientes, las técnicas anestésicas y el tipo de procedimiento.

Nosotros analizamos los procedimientos relacionados con la neuroanestesia buscando los parámetros demográfi- 
Tabla 1

Número absoluto y porcentaje de intervenciones neuroquirúrgicas realizadas en Cataluña durante los días de corte ANESCAT en 2003

\begin{tabular}{|l|c|c|c|}
\hline & Urgentes & Programadas & Total \\
\hline Cirugía de columna & 12 & 94 & 106 \\
\hline Craneotomías para la resección de tumor & 6 & $58 \%$ \\
\hline Craneotomías por hemorragia o trauma & 18 & 1 & 64 \\
\hline $\begin{array}{l}\text { Craneotomías para aneurismas o cirugía de } \\
\text { malformaciones arterio-venosas cerebrales (MAV) }\end{array}$ & $24 \%$ & 19 \\
\hline Derivaciones ventrículo-peritoneales & 3 & 4 & 6 \\
\hline Otros & 12 & 4 & $7 \%$ \\
\hline Total & 53 & 212 & 63 \\
\hline
\end{tabular}

Tabla 2

Porcentaje de intervenciones neuroquirúrgicas (cirugía de columna, craneotomías y derivaciones ventrículoperitoneales) en Norteamérica ${ }^{1}$ comparada con Cataluña

\begin{tabular}{|l|c|c|}
\hline & Norteamérica & Cataluña \\
\hline Cirugía de columna & $64 \%$ & $40 \%$ \\
\hline Craneotomías & $24 \%$ & $34 \%$ \\
\hline Derivaciones ventrículo-peritoneales & $5 \%$ & $3 \%$ \\
\hline
\end{tabular}

cos, el tipo de intervención, la duración de los procedimientos y las características de la recuperación postanestésica. Exponemos los resultados de forma descriptiva como número total y porcentajes respecto del total.

\section{Resultados}

Fueron recogidos un total de 23.236 cuestionarios. Los procedimientos neuroanestésicos fueron 265, que representa un 1,28\% del total de la actividad anestésica. Estos procedimientos se realizaron en un total de 28 hospitales: 12 públicos, con un total del $73,6 \%$ de los procedimientos, y 16 hospitales privados en los que se realizó el $26,4 \%$ de los procedimientos. Del total de procedimientos neuroanestésicos, $24(9,1 \%)$ se realizaron en la población menor de 15 años.

Estos datos permiten extrapolar que en el año 2003 en Cataluña se realizaron 6.909 procedimientos neuroquirúrgicos (95\% IC 6022-7847).

La edad media de los pacientes intervenidos fue de 48 años (IC 95\% 45,5-50,6). Un 52\% eran hombres y un $48 \%$ mujeres.
La puntuación en la escala de riesgo anestésico ASA fue: ASA I $22 \%$, ASA II 30,2\%, ASA III 31\%, ASA IV-V $16,9 \%$.

Los procedimientos programados representaron el $79,7 \%$ y el resto $(20,3 \%)$ fueron procedimientos urgentes.

El porcentaje de intervenciones según el tipo de cirugía se encuentra reflejado en la tabla 1 . La cirugía de columna fue la más frecuente, representando un 40,1\% del total de procedimientos neuroquirúrgicos.

Las craneotomías por hemorragia o trauma representaron el 7,2\% del total de las intervenciones de neurocirugía. A destacar que éstas constituyeron el 34,6\% de los procedimientos urgentes.

La duración media de la anestesia (incluyendo el tiempo quirúrgico) fue de $150 \pm 108 \mathrm{~min}$. La duración media de las craneotomías para la resección de tumor fue de $287 \pm 95 \mathrm{~min}$.

Los pacientes fueron controlados postoperatoriamente en una sala de recuperación convencional $(55,8 \%)$, en una unidad de cuidados monitorizados $(19,2 \%)$ o en una unidad de cuidados intensivos $(24,9 \%)$. Los pacientes sometidos a craneotomía fueron admitidos mayoritariamente en una en una Unidad de Cuidados Intensivos (41,6\%) o en una 
Unidad de Vigilancia monitorizada (33,7\%), siendo el resto $(24,7 \%)$ atendidos en sala de recuperación convencional tras pasar varias horas en la Unidad de Recuperación Postanestésica.

\section{Discusión}

La anestesia para neurocirugía en Cataluña constituía el $1,28 \%$ del número total de intervenciones quirúrgicas con anestesia, siendo este porcentaje muy similar al reportado por la encuesta realizada en Italia en 1999. En el estudio italiano se describió un total de 12.263 procedimientos anestésicos siendo el porcentaje de neuroanestesias del $2,6 \%{ }^{6}$.

Según la encuesta ANESCAT, las intervenciones neuroquirúrgicas más frecuentemente realizadas en Cataluña en 2003 fueron (en orden descendente) la cirugía de columna, las craneotomías para resección de tumor, craneotomías por traumatismo y las derivaciones ventrículo-peritoneales. Cabe destacar que estos datos coinciden con los referidos en la encuesta realizada en 1999 entre los neurocirujanos de Norteamérica y reportada por la Asociación Americana de Cirujanos Neurológicos (AANS) $^{1}$ (Tabla 2). Diagnosticar cuanto antes la aparición de complicaciones postoperatorias, para disminuir la morbimortalidad, es una de las características diferenciales de la neurocirugía ${ }^{5}$. Tras haber sido sometido a una craneotomía, los pacientes suelen quedar bajo vigilancia monitorizada o ingresan en una unidad de cuidados intensivos hasta el día siguiente a la intervención. La vigilancia monitorizada (overnight intensive care) ${ }^{2}$ se suele realizar en la zona de reanimación postanestésica, lo que conlleva una disminución de los costes respecto al ingreso en UCI. Además el disponer de esta posibilidad suele disminuir el número de cancelaciones de cirugía programada, debidas a la falta de disponibilidad de camas libres en UCI. Este último recurso quedaría limitado a cierto tipo de cirugía como intervenciones en fosa posterior y cirugía complicada.

Es importante conocer las necesidades de cuidados postoperatorios de los pacientes neuroquirúrgicos y saber la práctica habitual en nuestro medio. Cerca de un $45 \%$ de los pacientes requirieron vigilancia monitorizada o UCI durante, al menos, las primeras 24 horas postoperatorias. Esto representa que alrededor de 3.000 pacientes al año necesitan de ese recurso tras intervenciones neuroquirúrgicas.

Los resultados de la encuesta ANESCAT nos permiten saber que la práctica de la neuroanestesia en Cataluña es comparable en cuanto a volumen de pacientes y a número de cirugías realizadas con mayor frecuencia a otros países de Europa y Norteamérica ${ }^{4}$.

\section{Conclusiones}

Alrededor de siete mil procedimientos neuroanestésicos fueron realizados en Cataluña en el año 2003. Las cirugías de columna y las craneotomías para resección de tumores fueron las intervenciones más frecuentemente realizadas. Los pacientes con craneotomías fueron remitidos principalmente a Unidades de Cuidados Intensivos o áreas de Vigilancia Monitorizada durante el postoperatorio inmediato.

\section{Bibliografía}

1. American Association of Neurological Surgeons: National Neurosurgical Statistics, 1999 Procedural Statistics. [citado el día 16 de junio de 2006]. Disponible en http: //www.neurosurgerytoday.org.

2. Aps C. Surgical critical care: the overnight intensive recovery (OIR) concept. Br J Anesth 2004; 92: 164-167.

3. Clergue, F., Auroy, Y., Péquignot, F., Jougla, E., Lienhart, A., Laxenaire, M.C.: French survey of anaesthesia in 1996. Anesthesiology 1999; 91:1509-1520.

4. Fred, G., Barker, II., William, T.. Curry, Jr., Carter, B.S.: Surgery for primary supratentorial brain tumors in the United States, 1988 to 2000: The effect of provider caseload and centralization of care. Neuro-oncology January 2005.

5. Kelly, D.F.: Neurosurgical postoperative care. Neurosurg Clin N Am 1994; 5: 789-810

6. Peduto, V.A., Chevallier, P., Casati, A.: A multicenter survey of anesthesia practice in Italy, Minerva Anestesiol. 2004; 70: 473-491.

7. Sabaté, S., Canet, J., Muñoz, S., Castillo, J., Lucas, M., Mayoral, V.: Epidemiología de la anestesia en Cataluña en 2003. Med Clin (Bart). 2006;126 Supl 2: 13-18.

Hurtado, P.; Valero, R.; Fernández, S.; Alcón, A.; Fábregas, N.; Grupo ANESCAT: Intervenciones neuroquirúrgicas en Cataluña. Resultados de una encuesta anestésica durante 2003. Neurocirugía 2007; 18: 492-495.

Correspondencia postal: Dra. Neus Fábregas. Servicio de Anestesiología. Hospital Clinic de Barcelona. Villarroel 170. 08036 Barcelona 